\title{
Qubits and two-level atom states in representation of Malevich's squares
}

\author{
Vladimir Chernega ${ }^{1,{ }^{*}}$ and Olga Man'ko ${ }^{1,2}$ \\ ${ }^{1}$ Lebedev Physical Institute, Russian Academy of Sciences, 119991 Moscow, Russia \\ ${ }^{2}$ Bauman Moscow State Technical University, 105005 Moscow, Russia
}

\begin{abstract}
New description of two-level atom states as well as spin-1/2 states by means of standard probability distribution is constructed. It is used as alternative of wave functions and density operators. The quantum suprematism picture of the states in terms of Malevich's squares triada is presented.
\end{abstract}

The states of quantum systems are described by wave functions and density matrices. The complex wave function of the system corresponds to state vectors $|\psi\rangle$ belonging to a Hilbert space of the system states. The density matrix corresponds to the density operator $\varrho$ acting in the Hilbert space. For example, the state vector $|\psi\rangle$ of a two-level atom state (also spin-1/2 state) has two complex components $\psi_{1}$ and $\psi_{2}$ satisfying the normalization condition $\left|\psi_{1}\right|^{2}+\left|\psi_{2}\right|^{2}=1$. Recently new representation of quantum states (tomographic probability representation) was suggested for systems with continious variables (like quantum oscillator) [1] and for systems with discrete variables like spin-systems [2,3]. In this representation the states are associated with standard probability distributions like states in classical statistical mechanics.

The aim of this paper is to present the probability representation on example of qubit states. We use the simplest version of the tomographic probability representation where the probability distributions which are associated with two-level atom states are probabilities of dichotomic random variables. Such variables correspond to probabilities of coin positions "up" and "down". The geometrical picture illustrating the qubit states was recently [4] presented in the form of triada of Malevich's squares (black, red and white). The triada describes all possible pure states with state vector $|\psi\rangle$ and all mixed states with the density operator $\hat{\varrho}$.

The construction of probability representation is based on using eigenstates of the Pauli matrices with the corresponding density matrices of the form

$$
\varrho_{x}=\left(\begin{array}{ll}
1 / 2 & 1 / 2 \\
1 / 2 & 1 / 2
\end{array}\right), \varrho_{2}=\left(\begin{array}{cc}
1 / 2 & -i / 2 \\
i / 2 & 1 / 2
\end{array}\right), \varrho_{z}=\left(\begin{array}{ll}
1 & 0 \\
0 & 0
\end{array}\right)
$$

According to Born rule for arbitrary state of spin-1/2 system with density matrix $\hat{\varrho}$ the probabilities to have in this state spin projection on the axis $x, y$, and $z$ equal $+1 / 2$,

\footnotetext{
* Corresponding author: vchernega @gmail.com
} 
respectively reads: $p_{1}=\operatorname{Tr}\left(\varrho \varrho_{x}\right), p_{2}=\operatorname{Tr}\left(\varrho \varrho_{y}\right), p_{3}=\operatorname{Tr}\left(\varrho \varrho_{z}\right) . \quad$ In view of this the matrix $\$ \backslash$ rho $\$$ can be expressed in terms of the three probabilities, i.e.

$$
\varrho=\left(\begin{array}{cc}
p_{3} & p_{1}-\frac{1}{2}-i\left(p_{2}-1 / 2\right) \\
p_{1}-\frac{1}{2}+i\left(p_{2}-1 / 2\right) & 1-p_{3}
\end{array}\right) .
$$

One can see that this matrix satisfies all the properties of the density matrix: $\varrho=\varrho^{+}$, $\operatorname{Tr} \varrho=1$ and eigenvalues of the matrix are non-negative which means that the probabilities $0 \leq p_{1}, p_{2}, p_{3} \leq 1$ must satisfy the additional inequalities due Sylvester's criterion

$$
\left(p_{1}-1 / 2\right)^{2}+\left(p_{2}-1 / 2\right)^{2}+\left(p_{3}-1 / 2\right)^{2} \leq \frac{1}{4} .
$$

This inequality means that the spin-1/2 projections on $\mathrm{x}, \mathrm{y}, \mathrm{z}$-axis are not independent. Thus the states of the qubit (spin-1/2 or two-level atom system) are described by the probability vector $\vec{p}$ with three components $p_{1}, p_{2}, p_{3}$. It means that we constructed the invertable map of density matrix $\varrho$ into the probability vector $\vec{p}=\left(p_{1}, p_{2}, p_{3}\right)$. The state vector $|\psi\rangle$ in the probability representation is expressed in the form of vector with components $\psi_{1}$ and $\psi_{2}$

$$
\psi_{1}=\sqrt{p_{3}}, \psi_{2}=\sqrt{1-p_{3}}\left(\frac{p_{1}-1 / 2}{\sqrt{p_{3}\left(1-p_{3}\right)}}+i \frac{p_{2}-1 / 2}{\sqrt{p_{3}\left(1-p_{3}\right)}}\right),
$$

which are expressed in terms of three probabilities. We used the new formula

$$
\cos \varphi=\frac{p_{1}-1 / 2}{\sqrt{p_{3}\left(1-p_{3}\right)}}, \sin \varphi=\frac{p_{2}-1 / 2}{\sqrt{p_{3}\left(1-p_{3}\right)}} .
$$

For pure qubit states these expressions correspond to equality in Eq.(3). The representation of density matrix $\varrho$ in quantum suprematism picture means that we use three squares constructed on triangle with vertices A, B, C. The vertices A, B, C are situated on sides of equilateral triangle with side length equal to $\sqrt{2}$. The length $y_{k}$ of the $\mathrm{k}$-th side of the triangle $\mathrm{A}, \mathrm{B}, \mathrm{C}$ is expressed in terms of probabilities $p_{1}, p_{2}, p_{3}$ as follows: $y_{k}=\left[2 p_{k}^{2}+2 p_{k+1}+2 p_{k} p_{k+1}-4 p_{k}-2 p_{k+1}+2\right]^{1 / 2}$.

The sum of the areas of three Malevich's squares is

$$
S\left(p_{1}, p_{2}, p_{3}\right)=2\left[3+2\left(p_{1}^{2}+p_{2}^{2}+p_{3}^{2}\right)-3\left(p_{1}+p_{2}+p_{3}\right)+p_{1} p_{2}+p_{2} p_{3}+p_{3} p_{1}\right] .
$$

There is one-to-one correspondence of the qubit state and the triada of Malevich's squares [4]. Maximal value of the areas equals due to inequality (3) to 3 . The minimal value equals $3 / 2$. For classical states of three coins for which we have no restriction on probabilities (3) the maximal value of Malevich's squares area equals 6 . This difference corresponds to presence of quantum correlations of spin-1/2 projections on axes $\mathrm{x}, \mathrm{y}, \mathrm{z}$ for quantum system (qubit). The correlations are absent for classical system of three coins. The developed approach to replace the usual description of quantum states by state vectors and density matrices by probabilities can be generalized for arbitrary quantum systems.

\section{References}

1. S. Mancini, V. I. Man'ko, and P. Tombesi, Phys. Lett. A, 213, 1 (1996)

2. V. V. Dodonov, and V. I. Man'ko, Phys. Lett. A, 229, 335 (1997)

3. V. I. Man'ko, and O. V. Man'ko, J. Exp. Theor. Phys., 85, 430 (1997)

4. V. N. Chernega, O. V. Man'ko, and V. I. Man'ko,. J. Rus. Las. Res. , 38, 141 (2017) 